\title{
Quantitative factors to consider during IR inspections of Power Transformers \\ by Roderick Thomas
}

The Snell Group, Tyn-y-Coed, Glynhir Road, Pontardulais, Swansea, SA4 PX, UK, rthomas@thesnellgroup.com

\section{Abstract}

The use of non-contact infrared patterns and measurements to indicate overall machine or component condition continues to grow both in importance and application. The ability to monitor condition and predict impending failure when used as part of a condition based maintenance strategy can enhance reliability, reduce catastrophic failure and ensure maximum performance. Transformers are machines that can benefit directly from the application of infrared thermography firstly on a qualitative basis via pattern recognition and adopting infrared tools such as isotherms and saturation palettes and secondly on a quantitative basis adopting IR fusion of both thermal and digital images, multiple emissivity recognition, infrared trending and reporting. For example pattern recognition of the thermal images can reveal the following transformer problems:

- External and internal connection problems to bushings.

- Overheating load tap changers.

- Cooling tubes blocked, restricted or lateral gradient patter indicating transformer not level.

- Breakdown of surge protection.

$\bullet$

An understanding of the machine thermal dynamic and how this is influenced by various machine and environment factors is also considered. One of the advantages of this technology is that it can be adopted across varying engineering disciplines such as mechanical and electrical and that at a quantitative level the information can be analysed and condition of particular components predicted. It is anticipated that as more and more of these infrared devices are introduced the popularity and applications will also increase resulting ultimately in improved economic performance of transformers. This paper will discuss the current best practices for using thermography for condition monitoring of utility assets, guidelines for success and an overview of mistakes that are commonly made.

Key words: Condition monitoring, predictive failure, portable infrared cameras, noncontact temperature measurement, qualitative and quantitative thermography, heat transfer and trending. 
http://dx.doi.org/10.21611/qirt.2008.06_08_08 